\title{
ICRF mode conversion in three-ion species heating experiment and in flow drive experiment on the Alcator C-Mod tokamak
}

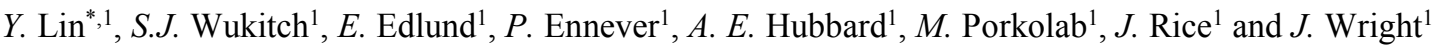 \\ ${ }^{1}$ MIT Plasma Science and Fusion Center, Cambridge, MA, USA
}

\begin{abstract}
In recent three-ion species (majority D and $\mathrm{H}$ plus a trace level of ${ }^{3} \mathrm{He}$ ) ICRF heating experiments on Alcator C-Mod, double mode conversion on both sides of the ${ }^{3} \mathrm{He}$ cyclotron resonance has been observed using the phase contrast imaging (PCI) system. The MC locations are used to estimate the species concentrations in the plasma. Simulation using TORIC shows that with the ${ }^{3} \mathrm{He}$ level $<1 \%$, most RF power is absorbed by the ${ }^{3} \mathrm{He}$ ions and the process can generate energetic ${ }^{3} \mathrm{He}$ ions. In mode conversion (MC) flow drive experiment in $\mathrm{D}\left({ }^{3} \mathrm{He}\right)$ plasma at $8 \mathrm{~T}$, MC waves were also monitored by PCI. The MC ion cyclotron wave (ICW) amplitude and wavenumber $\mathrm{k}_{\mathrm{R}}$ have been found to correlate with the flow drive force. The MC efficiency, wave-number $k$ of the MC ICW and their dependence on plasma parameters like $T_{\mathrm{e} 0}$ have been studied. Based on the experimental observation and numerical study of the dispersion solutions, a hypothesis of the flow drive mechanism has been proposed.
\end{abstract}

\section{Introduction}

Alcator C-Mod is a compact ( $a=0.22 \mathrm{~m}$ and $R=0.68 \mathrm{~m}$ ) tokamak running at high field $\left(B_{\mathrm{t} 0}<8.1 \mathrm{~T}\right)$ and high current $\left(I_{\mathrm{p}}<1.6 \mathrm{MA}\right)$. The ICRF system on Alcator CMod is able to provide reliable heating power up to $6 \mathrm{MW}$ from three antennas: D antenna $(80 \mathrm{MHz}), \mathrm{E}$ antenna $(80.5 \mathrm{MHz})$ and $\mathrm{J}$ antenna $(78 \mathrm{MHz})$.

Recently we have carried out the 3-ion species ICRF heating experiment (Section 2) and further investigated the mode conversion (MC) flow drive experiment in D${ }^{3}$ He plasmas (Section 3). In both experiments, we used the phase contrast imaging (PCI) diagnostic to detect the RF waves. In the 3 -ion species heating, the PCI observation of double mode conversion has been used to estimate the species concentration, thus providing the input parameters for TORIC wave simulation. In the flow drive experiment, PCI measured wave amplitude and $k_{\mathrm{R}}$ together with the numerical solutions of hot-plasma full-wave dispersion equations and TORIC simulation have been used to form a hypothesis of flow drive mechanism.

\section{Double mode conversion in 3-ion species ICRF heating experiments}

Three-ion species ICRF heating was discovered inadvertently on JET and the first controlled experiment was performed on Alcator C-Mod [1]. On both tokamaks, the enhanced heating effectiveness was confirmed and energetic ions were observed. In this paper, we discuss the feature of double mode conversion in the Alcator C-Mod experiment.

Effective ion heating happens when the ${ }^{3} \mathrm{He}$ level is so small that the ${ }^{3} \mathrm{He}$ ion cyclotron layer is located closely in the vicinity of two hybrid layers on both the high-fieldside (HFS) and the low-field-side (LFS). Near a hybrid layer $n_{\|^{2}}=S$, where $S$ is the first component of the dialectic tensor in Stix's notation and $\mathrm{n}_{\|}$is the component of the index of refraction parallel to the magnetic field, mode conversion from the fast wave (FW) to the ion Bernstein wave (IBW) and ion cyclotron wave (ICW) can occur. Moreover, the left-polarization component of the fast wave is greatly enhanced in the region. At a low ${ }^{3} \mathrm{He}$ level, the distance between the IC layer and the two hybrid layers are both sufficiently small so that the Doppler broadened IC layer can interact with the fast wave near the hybrid layers and result in strong power absorption to ${ }^{3} \mathrm{He}$ ions.

To carry out the experiment, we need to know the precise value of the species concentrations: $X\left[{ }^{3} \mathrm{He}\right]=$ $n_{\mathrm{He} 3} / n_{\mathrm{e}}$ and $X[\mathrm{H}]$. Fortunately, we can estimate the species concentrations between plasma shots from the PCI observation of the RF waves. PCI has been used to study different mode conversion scenarios on Alcator C-Mod, including ${ }^{3} \mathrm{He}-\mathrm{H}$ [2], $\mathrm{D}(\mathrm{H}), \mathrm{D}\left({ }^{3} \mathrm{He}\right)$ [3]. A detailed numerical study has been performed to understand the measured PCI wave signals. [4] In this 3-ion ICRF heating experiment, we successfully used PCI to monitor the species concentration based on the observation of double mode conversion.

In Fig. 1-(b), the amplitude of the PCI signal at the $\mathrm{RF}$ frequency is plotted vs. the major radius $\mathrm{R}$ and time $\mathrm{t}$. Two MC regions are clearly visible on the LFS and HFS of the ${ }^{3} \mathrm{He}$ cyclotron resonance, respectively: One at $R \approx$ $0.65 \mathrm{~m}$, the other at $0.71 \mathrm{~m}$, on HFS and LFS sides of the the ${ }^{3} \mathrm{He}$ IC layer at $R \approx 0.69 \mathrm{~m}\left(B_{\mathrm{t} 0}=7.83 \mathrm{~T}\right.$ and $\mathrm{RF}$ frequency $78 \mathrm{MHz}$ ).

\footnotetext{
Corresponding author: ylin@psfc.mit.edu
} 
Increasing $X[\mathrm{H}]$ would move both hybrid layers toward the HFS, and increasing $\left.X{ }^{3} \mathrm{He}\right]$ would separate the two layers farther apart. As a result, both $X[\mathrm{H}]$ and $X\left[{ }^{3} \mathrm{He}\right]$ can be uniquely determined from the two MC locations. For this particular plasma shot, we estimate that $X\left[{ }^{3} \mathrm{He}\right] \approx$ $0.9 \%, X[\mathrm{H}] \approx 65 \%$, assuming that all the remaining ions (mostly D plus small amount of impurities) have $A / Z=2$.
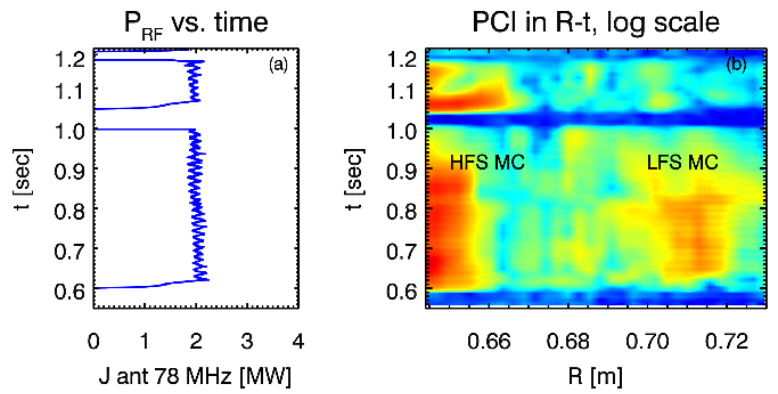

Fig. 1. (a) RF power; (b) Contour plot of PCI RF signal vs. $R$ - $t$.

In Fig. 2, we show the numerical solution of the full dispersion equation for the waves in the region. $[2,5]$. The HFS and LFS MC locations and cut-offs are also labelled. In this 3-ion heating scenario, while the MC waves provide direct electron heating, the important and unique feature is that the fast wave is strongly absorbed by the ${ }^{3} \mathrm{He}$ ions near the ${ }^{3} \mathrm{He}$ IC layer. Note that $\operatorname{Im}\left(k_{\perp}\right)$ of the fast wave in this region $(0.67 \mathrm{~m}<R<0.72 \mathrm{~m})$ is more than one order of magnitude larger than the FW branches at $R$ $<0.65 \mathrm{~m}$ and $R>0.74 \mathrm{~m}$.

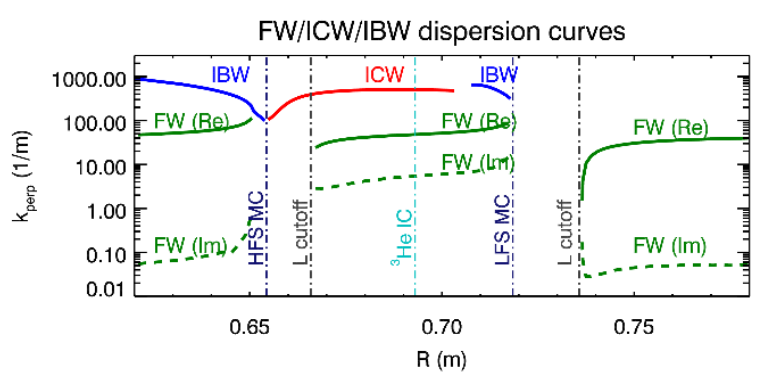

Fig. 2. Numerical solution of the full dispersion solution for the waves. For the fast wave, both $\operatorname{Im}\left(k_{\perp}\right)$ and $\operatorname{Re}\left(k_{\perp}\right)$ are shown.

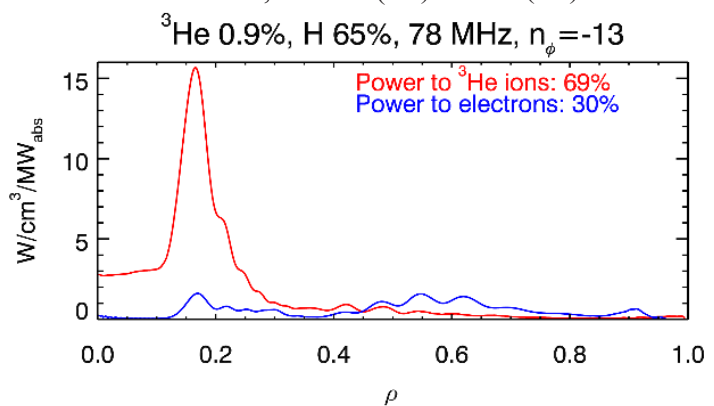

Fig. 3. Power deposition profiles from TORIC simulation.

The strong FW ion heating has been confirmed by 2-D full wave simulation using TORIC. As shown in Fig. 3 , about $70 \%$ of the RF power is absorbed by ${ }^{3} \mathrm{He}$ ions via localized strong ion absorption near axis. In contrast, the electron absorption via the fast wave and MC wave is broad and weak. For some other plasmas in the experiment with higher $\left.X{ }^{3} \mathrm{He}\right]$, e.g., $~ 3 \%$, the simulation shows that most RF power is to the electron heating via mode conversion.

\section{ICRF wave measurements in $\mathrm{D}\left({ }^{3} \mathrm{He}\right)$ flow drive experiments}

\subsection{Experimental observation}

Mode conversion flow drive has been studied extensively on Alcator C-Mod [6, 7]. A detailed scaling law has been obtained vs. main plasma parameters and RF parameters. However, in all the previous flow drive experiments, PCI was unavailable for direct RF wave measurement. In lack of directly supporting experimental evidence, we established a plausible correlation between the greatly enhanced rotation with RF power in $\mathrm{D}\left({ }^{3} \mathrm{He}\right)$ plasmas with $X\left[{ }^{3} \mathrm{He}\right]$ from $10 \%-15 \%$ and the MC process by the comparison with the intrinsic rotation in $\mathrm{D}(\mathrm{H})$ minority heating and by TORIC simulation. Lately, we have further investigated the MC flow drive experiment with an improved PCI system for RF wave measurement. This measurement has provided a more direct comparison of the wave physics and the driven rotation.
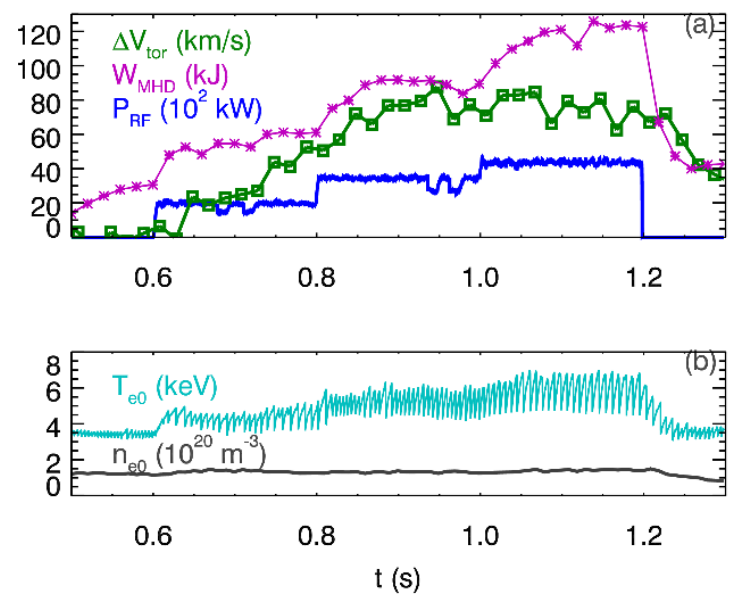

Fig. 4. Traces of parameters for a plasma in $\mathrm{MC}$ flow drive experiment: (a) $W_{\mathrm{MHD}}, \Delta V_{\mathrm{tor}}$, and $P_{\mathrm{RF}}$; (b) $T_{\mathrm{e} 0}$ and $n_{\mathrm{e} 0}$.

In Fig. 4, we compare the time traces of major plasma parameters from one of the 6 plasmas in the experiment. $I_{\mathrm{p}}=1.2 \mathrm{MA}, X\left[{ }^{3} \mathrm{He}\right] \sim 14 \%$, and $B_{\mathrm{t} 0}=7.99$ $\mathrm{T}$. The direction of $\mathrm{B}$ field is in the direction that is unfavourable for $\mathrm{H}$-mode on $\mathrm{C}$-Mod, i.e., $B \times \nabla B$ pointing away from the divertor. The plasma starts in L-mode confinement and enters I-mode at $\mathrm{t} \sim 0.9 \mathrm{sec}$ according to the signature of a weakly coherent mode in the plasma edge (not shown here) [8, 9]. In Fig. 4-(a), the change of the central toroidal rotation after the RF power is applied, $\Delta V_{\text {tor }}(t)=V_{\text {tor }}(t)-V_{\text {tor }}(t=0.6 \mathrm{~s})$, is shown together with the traces of the stored energy $W_{\text {MHD }}$ and total RF power $P_{\mathrm{RF}}$. The time traces of $T_{\mathrm{e} 0}$ and $n_{\mathrm{e} 0}$ are shown in Fig. 4(b). In this plasma shot, $P_{\mathrm{RF}}$ has three steps at $2 \mathrm{MW}, 3.5$ MW and 4.5 MW, each lasting for 0.2 second. It is evident that along with the stepping up of $P_{\mathrm{RF}}, W_{\mathrm{MHD}}$ and $T_{\mathrm{e} 0}$ both show definite increases in steps, even for the highest RF power step. In contrast, $\Delta V_{\text {tor }}$ rises rapidly at the first two $P_{\mathrm{RF}}$ steps but stops increasing at the last power step even if the plasma is in I-mode, in which the rotation is expected to rise from the intrinsic rotation drive from the pedestal region. [10] Such rapidly rising and saturating 
behaviour in $\Delta V_{\text {tor }}$ is similar for all other plasmas in this $\mathrm{MC}$ flow drive experiment. It has also been observed in some previous MC flow drive experiments in I-mode at high $P_{\mathrm{RF}}$ aiming at, but unsuccessfully, synergetic linear supposition of the I-mode intrinsic rotation and $\mathrm{MC}$ driven rotation. Note that the intrinsic rotation and the MC driven flow cannot be easily separated, especially in the Imode phase. To some extent, the root cause of the intrinsic rotation in I-mode can also be traced back to the ICRF heating. In this preliminary study, we only study the change of the total rotation vs. the RF.

As shown in Fig. 4, at the low to middle levels of $P_{\mathrm{RF}}, \Delta V_{\text {tor }}$ is strongly correlated with $W_{\text {mhd }}$ and $T_{\mathrm{e} 0}$, probably due to the common effect of the RF heating. However, the saturation of $\Delta V_{\text {tor }}$ at high $P_{\mathrm{RF}}$ (albeit with better plasma confinement and higher $T_{\mathrm{e} 0}$ ) indicates something more subtle is at work and PCI observation provides a clue.

In Fig. 5, PCI measured RF signal for this plasma is shown vs. $R$ - $t$ and vs. $k_{\mathrm{R}}-t$. Strong MC waves are detected for $t \approx 0.6-1.0 \mathrm{sec}$, but the MC signal becomes weaker in time and becomes nearly undetectable after $t>1.0 \mathrm{sec}$ although the RF power is higher. The MC wave is mostly at $k_{\mathrm{R}} \sim 3-7 \mathrm{~cm}^{-1}$ and the fast wave peak is at $k_{\mathrm{R}}<1 \mathrm{~cm}^{-1}$.
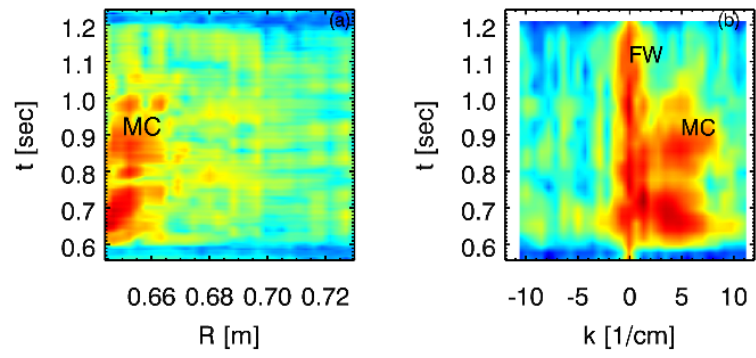

Fig. 5. Contour plot of the PCI RF signal (a) vs. $R$ - $t$; (b) vs. $k_{\mathrm{R}}-$ $t$. The RF power trace ( $\mathrm{J}$ antenna at $78 \mathrm{MHz}$ ) is in Fig.6-(b).

To better show the time evolution of the PCI observation, in Fig. 6 we compare the PCI observed FW amplitude and $\mathrm{MC}$ wave amplitude (mostly from the MC $\mathrm{ICW}$ ). The MC ICW is from the $\mathrm{J}$ port antenna (also shown in Fig. 5), and the FW is from E port antenna at (80 MHz). In Fig.6-(a), the PCI measured FW signal appears to broadly follow the applied RF power level. This behaviour is expected because the PCI signal $\propto|E|^{2}$. On the other hand, in Fig. 6-(b), the PCI MC ICW signal is very different. It starts with a quite high level at the beginning of the RF pulse ( $P_{\mathrm{RF}} \sim 1 \mathrm{MW}$ for J antenna) at a moderate $T_{\mathrm{e} 0} \sim 3 \mathrm{keV}$, and with the stepping up of $P_{\mathrm{RF}}$ and the rise of $T_{\mathrm{e} 0}$, the $\mathrm{MC}$ wave amplitude level actually drops precipitously. By the time at $t>1.0 \mathrm{sec}$ when the RF power reached the $2 \mathrm{MW}$ level and the peak electron temperature reaches $T_{\mathrm{e} 0} \sim 6 \mathrm{keV}$, the MC amplitude in PCI has become less than $5 \%$ of its peak level at $t=0.65$ sec. In Fig. 4-(a), it is shown that the rotation increases rapidly when the RF power is at the low to middle level but the rotation speed levels off at the highest RF power level.

As shown in Ref. [4], the level of the PCI signal has been found to be very difficult to calculate from given plasma parameters and RF parameters. Many factors prevent us from obtaining quantitative interpretation of the PCI RF measurement. For instance, PCI takes a snapshot of a cross-section far away from the antenna but the MC process is essentially a 3-D problem. In this study, we limit our scope only to the trend of the PCI signal (but not the absolute level) vs. the observed rotation. In Fig. 7, we show the time traces of $n_{\mathrm{e} 0} \times d V_{\text {tor }} / d t$, i.e., a proxy of the driving force, vs. $\log _{10}$ (PCI MC amplitude) for all 6 shots in the experiment. For the entire data set, the correlation between these two quantities is about 0.39 , while the correlation between the drive force and other parameters (e.g., $T_{\mathrm{e} 0}$ and PCI FW amplitude) are close to zero $(<0.1)$. Fig.7 shows that the correlation possibly exists and persists for each and all plasma shots.
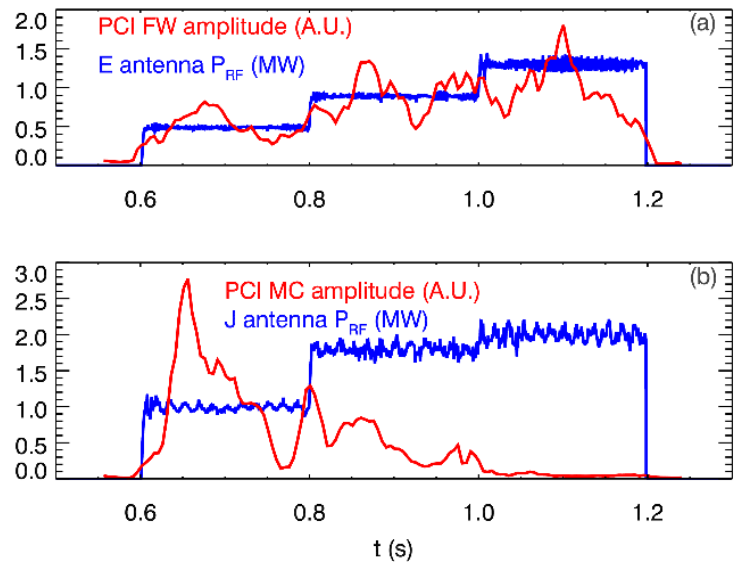

Fig. 6. (a) PCI FW signal time trace vs. $P_{\mathrm{RF}}$ (E antenna, 80 $\mathrm{MHz}$ ); (b) PCI MC wave signal trace vs. $P_{\mathrm{RF}}(\mathrm{J}$ antenna, $78 \mathrm{MHz})$.

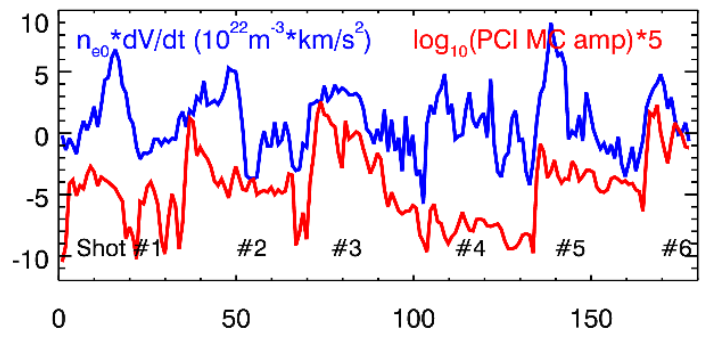

Fig. 7. Scaled $\log _{10}(\mathrm{PCI}$ MC amplitude) vs. the proxy rotation drive force. The time traces are from consecutive 6 plasma shots in the experiment.

\subsection{Dispersion equation solution and $T_{\mathrm{e} 0}$ dependence}

In this exploratory experiment, we were aiming at finding a correlation with the PCI measurement although it was not specific what to expect. Can the correlation shown in Fig. 7 indicate possible causality? In order to further understand whether the correlation would indicate possible causality, we have studied the dispersion solution of the MC process for these plasmas, especially that of the MC ICW.

As shown in Ref. [2, 3, 5], the MC ICW solution can be obtained from the full dispersion equation combined with the poloidal field induced wave-vector $k$ transformation equation. This approach, albeit imprecise, is better in pointing out the possible physics mechanisms than full-wave simulations using TORIC or AORSA. In Fig. 8-(a), the dispersion solution of all three waves: the 
fast wave, the MC IBW and ICW are plotted vs. major radius for the case where $n_{\mathrm{e} 0}=1.5 \times 10^{20} \mathrm{~m}^{-3}$ and $T_{\mathrm{e} 0}=3$ $\mathrm{keV}$. For the MC ICW, both real and imaginary components of the complex $k_{\perp}$ are shown. Note both $\operatorname{Re}\left(k_{\perp}\right)$ and $\operatorname{Im}\left(k_{\perp}\right)$ of the MC ICW increases along with the propagation path toward the LFS. Its $k_{\|}$also increases as constrained by the $k$-vector transformation for a given $k_{\phi}$. A normalized power deposition of the MC ICW, calculated from the ratio of $\operatorname{Re}\left(k_{\perp}\right)$ and $\operatorname{Im}\left(k_{\perp}\right)$, is in Fig. 8 -(b), where the peak deposition is at about $0.8 \mathrm{~cm}$ from the MC layer and nearly no more power is left after propagating for $2 \mathrm{~cm}$ in the direction perpendicular to $\mathrm{B}$ field (equivalently $\sim 25 \mathrm{~cm}$ in the direction parallel to B). This is consistent with the PCI observation in Fig. 5-(b).
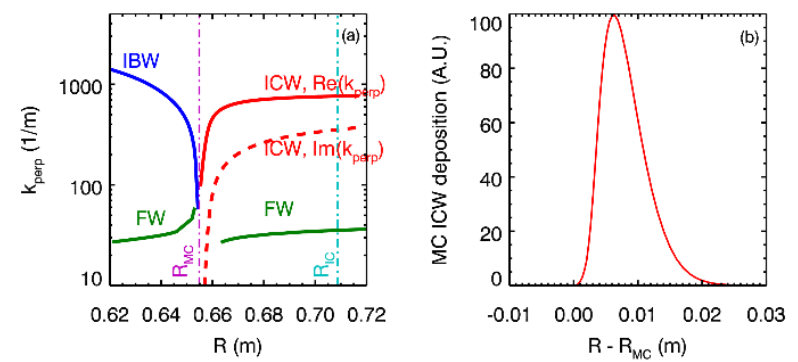

Fig. 8. (a) Wave solution from hot-plasma full-wave dispersion equation; (b) Deposition profile of the MC ICW.

In Fig. 9-(a) and 9-(b), we plot the result of a $T_{\mathrm{e} 0}$ scan of the dispersion solution. Both $R e\left(k_{\perp}\right)$ and $k_{\|}$of ICW decrease quickly vs. $T_{\mathrm{e} 0}$. Since geometrically PCI $k_{\mathrm{R}} \sim k_{\perp}$, the numerical result agrees with the PCI observation in Fig. 5-(c), where $k_{\mathrm{R}}$ of the PCI MC signal starts at $k_{\mathrm{R}} \sim 7$ $\mathrm{cm}^{-1}$ and then decreases vs. time to $k_{\mathrm{R}} \sim 3 \mathrm{~cm}^{-1}$, along with the rise of plasma $T_{\mathrm{e} 0}$. In other words, the MC ICW is absorbed at larger $k_{\perp}$ and $k_{\|}$at lower $T_{\mathrm{e} 0}$. In addition, Fig. 9-(c) shows that there is also more RF power absorption via the MC ICW (plus a small portion from the FW) according to the TORIC calculation $\left(n_{\phi}=13\right)$.
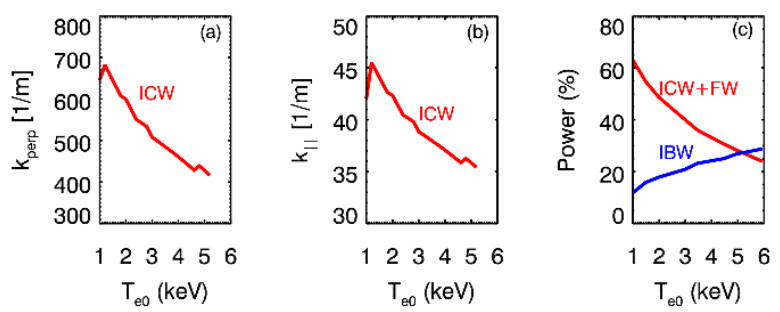

Fig. 9. Dispersion solution and $\mathrm{MC}$ power vs. $T_{\mathrm{e} 0}$. (a). $R e\left(k_{\perp}\right)$; (b) $k \|$; (c) The fraction of total RF power to the MC ICW and the MC IBW from TORIC calculation.

In other words, the experimental result, dispersion solution and TORIC simulation together suggest that at lower $T_{\mathrm{e} 0}$, there is more RF power converted to the MC ICW and the MC ICW then deposits power at larger $k_{\|}$ and $k_{\perp}$ than at higher $T_{\mathrm{e} 0}$.

\subsection{Hypothesis on the flow drive mechanism}

Experimentally, we have observed that the PCI MC ICW amplitude is much larger at lower $T_{\mathrm{e} 0}$, so is the force driving the rotation. The correlation between these two observations appears meaningful. From the analysis in Section 3.2, we can explain the behaviour of the PCI signal from the mode conversion physics, but we still need to find a physical mechanism that may connect the $\mathrm{MC}$ waves and the force driving the rotation, i.e., $n_{\mathrm{e} 0} \times d V_{\mathrm{tor}} / d t$.

Note that a wave at larger wavenumber $k$ (i.e., slower velocity) would generally have large momentum per unit of power. A MC ICW with larger amplitude and at larger $k_{\|}$at the absorption would in theory mean a larger momentum transfer to the plasma (both ions and electrons) after its power has fully deposited. In addition, the MC process to ICW is up-down asymmetric due to poloidal field effect. [3] As a result, the MC ICWs above the mid-plane and below the mid-plane are at different $r / a$ locations in terms of origin, propagation and absorption. The MC process might work like a powerful "engine" that churns the core plasma and re-distributes momentum and then the plasma ultimately gains net momentum from the friction with the wall. This "engine" would be more powerful at lower $T_{\mathrm{e} 0}$ where more RF power is converted to the $\mathrm{MC} \mathrm{ICW}$ and at higher $k$, consistent with the observed dependence of the driving force.

We may find more supportive evidence from data mining and simulation, but the hypothesis needs to be tested in future experiments. For example, would the rotation continue rising if we can maintain a high level of PCI MC amplitude using moderate RF power and at moderate $T_{\mathrm{e} 0}$ ? Would the plasma rotate faster if not entering I-mode? After the closure of Alcator C-Mod in 2016, these questions can only be answered by future experiments on other devices.

\section{Summary}

Double mode conversion has been observed in the 3-ion ICRF heating scenario by PCI. Plasma composition can be accurately determined and used as input for full-wave simulation. In the $\mathrm{MC}$ flow drive experiment, the fast wave and $\mathrm{MC}$ waves have been detected directly for the first time and interesting correlation has been found between the PCI MC amplitude vs. the rotation drive.

This work was supported by US DoE Cooperative agreement DE-FC02-99ER54512 at MIT using the Alcator C-Mod tokamak, a DOE Office of Science user facility.

\section{References}

1. Y.O. Kazakov et al, Nature Phys. (19 June 2017) doi: $10.1039 /$ nphys4167.

2. E. Nelson-Melby et al, Phys. Rev. Lett. 90, 155004 (2003).

3. Y. Lin et al, Plasma Phys. Controlled Fusion 47, 1207 (2005).

4. N. Tsujii et al, Phys. Plasma 22, 082502 (2015).

5. E. F. Jaeger et al, Phys. Rev. Lett. 90, 195001 (2003).

6. Y. Lin et al, Phys. Rev. Lett. 101, 235002 (2008).

7. Y. Lin et al, Nucl. Fusion. 51, 063002 (2011).

8. D. G. Whyte et al, Nucl. Fusion 50, 105005 (2010).

9. A. E. Hubbard et al, Nucl. Fusion 52, 114009 (2012).

10. J. Rice et al, Phys. Rev. Lett. 106, 215001 (2011). 